Título de la Publicación:

Influence of ligand structure and molecular geometry on the properties of $d 6$ polypyridinic transition metal complexes

\title{
Autor:
}

Arias M., Concepcion J., Crivelli I., Delgadillo A., Diaz R., Francois A., Gajardo F., Lopez R., Leiva A.M., Loeb B.

\section{Datos de Publicación:}

Chemical Physics, Vol. 326, N¹, 54-70, 2006

Disponible en:

http://www.scopus.com/inward/record.url?eid=2-s2.0-

$33745486033 \&$ partnerID=40\&md5=1d6921f3f8aeb0cd615bc353a868bbb7 